\title{
Unexpected changes in direction of motion attract attention
}

\author{
Christina J. Howard And Alex O. Holcombe \\ University of Sydney, Sydney, Australia
}

\begin{abstract}
Under some circumstances, moving objects capture attention. Whether a change in the direction of a moving object attracts attention is still unexplored. We investigated this using a continuous tracking task. In Experiment 1 , four grating patches changed smoothly and semirandomly in their positions and orientations, and observers attempted to track the orientations of two of them. After the stimuli disappeared, one of the two target gratings was queried and observers reported its orientation; hence direction of the gratings' motion across the screen was an irrelevant feature. Despite the irrelevance of its motion, when the nonqueried grating had collided with an invisible boundary within the last $200 \mathrm{msec}$ of the trial, accuracy reporting the queried grating was worse than when it had not. Attention was likely drawn by the unexpected nature of these changes in direction of motion, since the effect was eliminated when the boundaries were visible (Experiment 2). This tendency for unexpected motion changes to attract attention has important consequences for the monitoring of objects in everyday environments.
\end{abstract}

The world is full of moving objects and people whose motions are complex and frequently changing. As pedestrians in the city, for example, we are confronted by many vehicles, each moving in its own fashion. In many sports, players are constantly starting, stopping, and changing direction. How do changes in motion affect our awareness of object properties? When a person or vehicle changes direction of motion, often it is because intentions have changed or a new goal has been acquired. It may be important to then reevaluate the situation - for example, to determine whether the object is now on course to intercept or collide with other things. Here we report evidence that changes in the direction of motion recruit visual attention.

Several visual characteristics appear to capture attention. For instance, the appearance of a new object among a background of old objects can capture attention (Enns, Austen, Di Lollo, Rauschenberger, \& Yantis, 2001; Yantis \& Hillstrom, 1994; Yantis \& Jonides, 1984), although these effects are sometimes attributable to the attentioncapture effects of luminance changes (see, e.g., Enns et al., 2001). Color changes also can capture attention (von Mühlenen, Rempel, \& Enns, 2005), as can an odd color in a search display (Horstmann, 2005; Johnson, Hutchison, \& Neill, 2001).

Differences in the motion of objects in a scene can also help to direct our attention. For instance, visual search is efficient when the target is defined by a particular direction of motion among an array of distractors all moving in a second direction (Ansorge, Scharlau, \& Labudda, 2006), and observers are able to perform visual search for different motion types (Horowitz, Wolf, DiMase, \& Klieger,
2007). Furthermore, even if distractors are moving in various directions, if these motions can all be attributed to self-motion then a target moving in a contrary fashion is conspicuous (Rushton, Bradshaw, \& Warren, 2007).

The presence of motion, or at least the onset of motion, among stationary objects appears to attract attention under some circumstances. For instance, Franconeri and Simons (2003) used an irrelevant-feature search to show that motion-defined singletons can capture attention. In their experiments, translating and looming stimuli captured attention in an array of otherwise stationary stimuli. Some experiments have suggested that the onset of motion (a special case of a change in motion, going from no motion to some motion) is particularly potent for capturing attention (Abrams \& Christ, 2003). The question of which aspects of moving stimuli capture attention is controversial, but it is clear that in many circumstances moving stimuli are potent attention-grabbers (Abrams \& Christ, 2003, 2005a, 2005b, 2006; Franconeri \& Simons, 2005).

To our knowledge no one has tested directly whether changes in motion can attract attention. Certainly, the pattern of changes in motion can be a powerful cue for the perception of animacy and causality (Michotte, 1963). Since perceiving animacy and causality is important, it might be adaptive for motion changes generally to attract attention. As mentioned above, when a person, animal, or vehicle changes direction of motion, their intentions have often changed, which is another reason to attend to these events. If the object is not animate, changes in direction of motion often occur as a result of new forces, such as a sudden gust of wind or the impact of another object. For these 
reasons, changes in direction of motion may attract attention. We show here that attention is attracted by certain changes in direction of motion that are not accompanied by an obvious cause.

In our experiments, observers continuously tracked the changing orientations of two moving objects in the presence of two similar objects. The effect of change in direction of motion after collisions with boundaries was assessed by examining accuracy of reporting the final orientation of one of the tracked objects. In Experiment 1, a change in direction of motion of the nonqueried object decreased accuracy in reporting the features of the queried object. These direction changes occurred as a result of collision with invisible boundaries; hence, they were not attributable to any visible feature on the screen and often may have been unanticipated by the participants. In Experiment 2, the boundaries were visible, and the effect of direction changes no longer occurred. We conclude that unexplained changes in motion direction attract attention.

\section{EXPERIMENT 1}

\section{Method}

Experiment 1 was designed to test whether a change in direction of motion facilitates awareness of stimulus features. Observers attempted to track the continuously changing orientations of two grating patches out of a total of four on the screen. To reflect the varying trajectories and object changes encountered in many realworld situations, the gratings changed in position and orientation in a semirandom manner.

A computer program written in Python using the Vision Egg library (Straw, 2008; www.visionegg.org) displayed an array of four sinusoidal luminance gratings against a mid-gray background on a 16-in. CRT screen refreshing at $60 \mathrm{~Hz}$. Observers viewed the display in a dimly lit room from a distance of $0.4 \mathrm{~m}$.

The luminance at the trough of each grating was less than $1 \mathrm{~cd} / \mathrm{m}^{2}$, at the peak it was $88 \mathrm{~cd} / \mathrm{m}^{2}$, and the spatial frequency was $3.90 \mathrm{cpd}$.
A circular mask windowed the grating patches and had a radius of $0.65 \mathrm{deg}$ of visual angle. A white circular fixation point with diameter of 0.42 deg was displayed at the center of the screen throughout the experiment.

Observers. Observers were 8 psychology staff members and 10 postgraduate students at Bristol University, plus 1 undergraduate and 1 staff member at the University of Sydney. Sixteen were naive to the purpose of the experiment, and 12 were nonexpert in participating in visual psychophysical experiments. All had normal or corrected-to-normal vision.

Procedure. Observers were given practice trials until they felt comfortable with the experiment, which usually required less than 10 trials. They then completed 105 trials each. Four invisible motionlimiting "boxes" measuring $10.98 \times 10.98 \mathrm{deg}$ were arranged with their innermost corners 3.14 deg away from the fixation point, one in each of the Cartesian quadrants. At the start of each trial, four gratings were displayed $10.88 \mathrm{deg}$ eccentric from fixation, each at the center of its respective box. At the start of each trial, two gratings were marked as targets by flashing squares, measuring $2.22 \times$ $2.22 \mathrm{deg}$, which flashed black-white for $4.2 \mathrm{sec}$ at $1 \mathrm{~Hz}$ (see Figure 1). On every trial, each grating had an equal chance of being chosen as one of the two targets, and each target had an equal chance of being queried at the end of the trial.

Immediately after the disappearance of the flashing squares, each grating underwent a period of smooth and quasirandom motion within its box and also changed smoothly and quasirandomly in its orientation (see Figure 2). At a random time between $4,250 \mathrm{msec}$ and $11,330 \mathrm{msec}$ after the start of motion, all gratings disappeared. One of the targets was postcued $300 \mathrm{msec}$ later. We chose to delay the appearance of the postcue by $300 \mathrm{msec}$ to reduce any possibility of observers "reading out" the final orientation from high-capacity iconic memory (Averbach \& Coriell, 1961; Coltheart, Lea, \& Thompson, 1974; Shooner, Tripathy, Bedell, \& Ögmen, 2010; Sperling, 1960) without paying attention to the targets prior to their disappearance. To postcue one of the targets, its quadrant was indicated with a black square that appeared near one of the four corners of the screen (subtending $2.2 \times 2.2 \mathrm{deg}$ and centered 15.2 deg horizontal and $13.1 \mathrm{deg}$ vertical from fixation). Observers attempted to report the last orientation of the grating that had previously occupied that box. This grating will be referred to as the queried target.

\section{Quadrants containing targets All four gratings change smoothly and Blank (300 msec) for tracking are marked randomly in position and orientation}

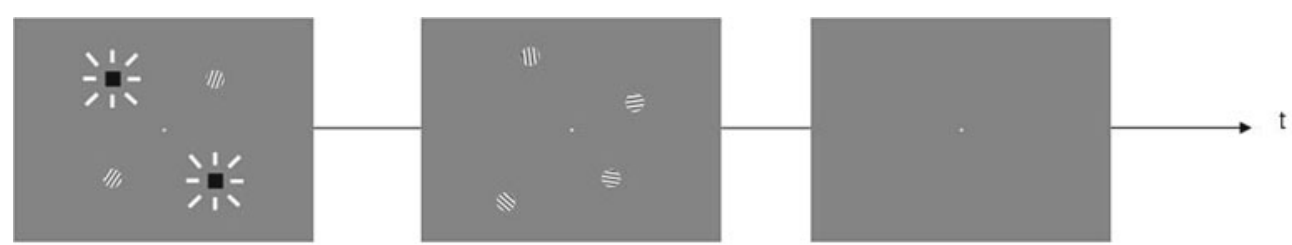

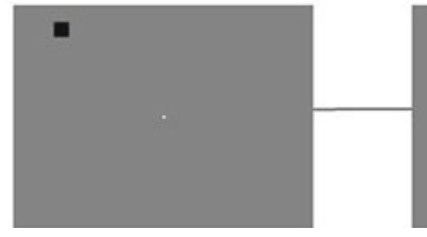

One of the targets is queried

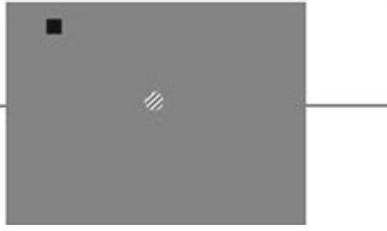

Report last orientation of queried grating

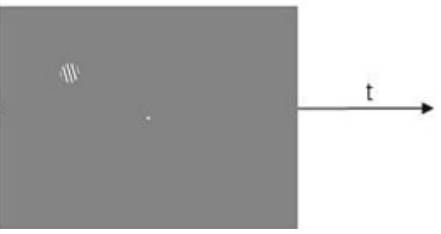

Feedback

Figure 1. Trial timeline. Observers adjusted the orientation of the response grating patch to match the last perceived orientation of the queried target. In the example depicted here, the observer reports an orientation of $\mathbf{- 4 0} \mathrm{deg}$. Given that the last orientation of the queried target was $18 \mathrm{deg}$, this represents an error of $\mathbf{- 5 8} \mathrm{deg}$. 


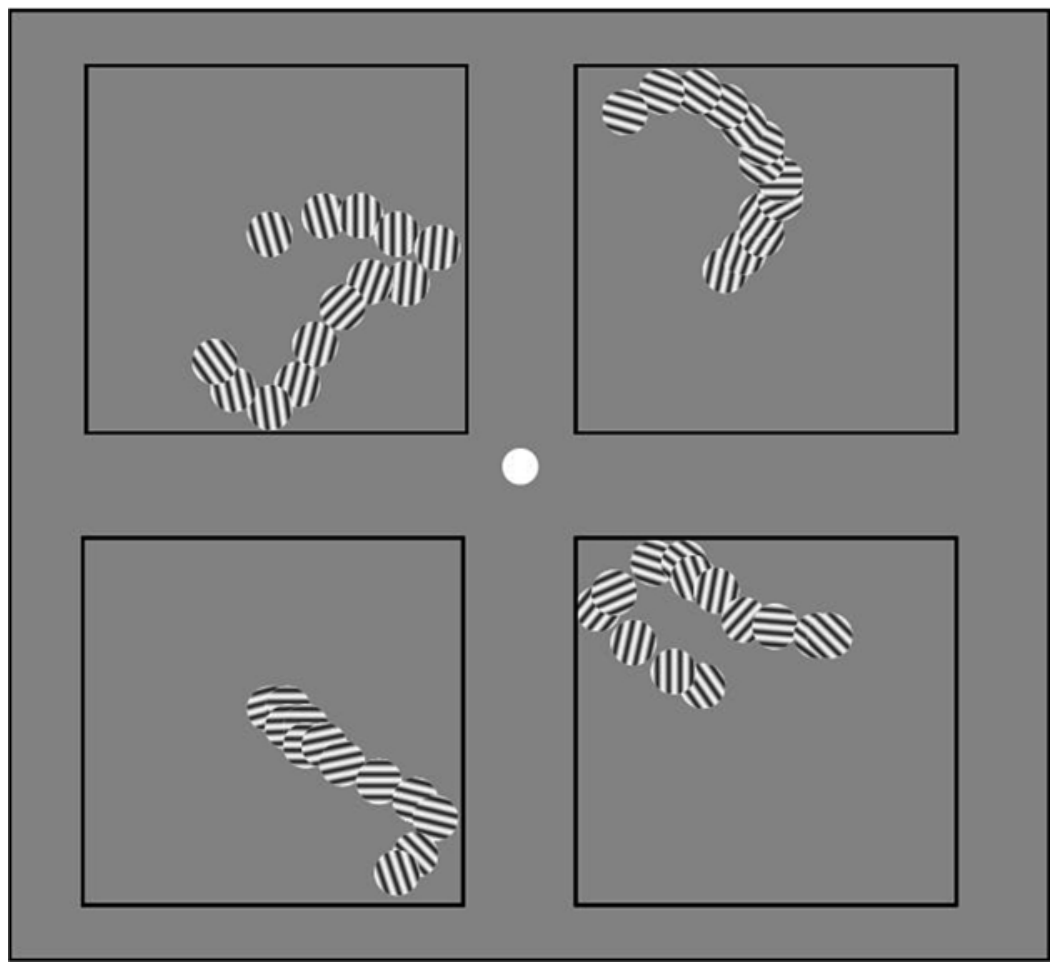

Figure 2. Trajectories of the four gratings in a typical trial. The various grating positions mark their locations every 330 msec. For the grating in the upper left quadrant, two "bounces" off box boundaries can be seen. In Experiment 1, the box boundaries were not visible; in Experiment 2, they were drawn as shown.

Observers used two keys to adjust the orientation of the response grating to match the last orientation of the queried target. The response grating, which was presented at fixation, was initially oriented vertically. However, it did not appear until the first frame $(16.67 \mathrm{msec})$ after observers began their keypress response, which they did by pressing one of the arrow keys, which rotated the response grating by a small step. Following the observer's response, the queried target was presented as feedback in its true final position and orientation.

Position trajectories of gratings. Each grating's trajectory within each box was independently generated by the following algorithm. Horizontal and vertical velocities were generated entirely independently. Starting horizontal and vertical velocities were chosen randomly and independently from a uniform distribution spanning -2.67 to $2.67 \mathrm{dps}$, excluding very slow speeds of less than $0.27 \mathrm{dps}$. Starting horizontal and vertical accelerations were randomly and independently allocated for each grating and were chosen from one of two values, one of which was negative and one of which was positive. For gratings that initially were set to a fast speed (greater than $1.33 \mathrm{dps}$ ), the possible accelerations were a pair of positive and negative values with magnitude of $3.20 \mathrm{deg} / \mathrm{sec}^{2}$. To prevent gratings from remaining still for a prolonged period, the possible accelerations were higher for slow gratings (less than $1.33 \mathrm{dps}$ ), either positive or negative $8.00 \mathrm{deg} / \mathrm{sec}^{2}$.

For each grating, there was a 1 in 20 chance of a reallocation of its acceleration on every frame. When this occurred, acceleration was randomly reassigned one of the two positive and negative values described above, depending on the current velocity. A maximum absolute value (speed limit) for horizontal and vertical velocities was set at $5.34 \mathrm{dps}$. If any grating reached the maximum speed, the acceleration direction was reversed, causing it to tend back toward slower velocities. The mean resulting speed generated by the algorithm was $3.1 \mathrm{dps}$.
Gratings were bounded within their boxes by checking whether each had come closer than $0.24 \mathrm{deg}$ to the edge of the boxes. If the location of a grating reached this value, the sign of the velocity along the offending dimension was reversed, causing it to apparently bounce off the boundary (the 0.24-deg gap with the boundary was not noticeable). For instance, if a grating approached within 0.24 deg of its upper boundary, the vertical velocity was reversed, with the horizontal velocity left unchanged.

Orientation trajectories of gratings. Orientations were set independently for each grating and independent of their position trajectories. Objects' starting orientations were set to random values. Starting angular velocities (change in orientation per unit of time) were set independently to a random value between $-30 \mathrm{dps}$ and $30 \mathrm{dps}$, excluding absolute values less than $6 \mathrm{dps}$, so that no gratings appeared to be not rotating when first presented. Starting angular accelerations were randomly and independently chosen from one of two values, one of which was negative and one of which was positive. For gratings with high angular velocity (greater than an absolute value of $12 \mathrm{dps}$ ), the possible accelerations were a pair of positive and negative values with magnitude of $36 \mathrm{~d} / \mathrm{sec}^{2}$. For gratings with slow angular velocity (less than an absolute value of $12 \mathrm{dps}$ ), the possible accelerations were higher, either positive or negative $108 \mathrm{deg} / \mathrm{sec}^{2}$.

For each grating, there was a 1 in 20 chance of a reallocation of angular acceleration on every frame. When this occurred, acceleration was randomly reassigned one of the two positive and negative values, depending on the current angular velocity, as described above. If the angular speed was below $12 \mathrm{dps}$ at any time, then the absolute value of the acceleration was increased from $36 \mathrm{deg} / \mathrm{sec}^{2}$ to $108 \mathrm{deg} / \mathrm{sec}^{2}$. The angular speed was not allowed to exceed $180 \mathrm{dps}$. If any grating reached this value, the direction of its acceleration was reversed, so that its speed would begin decreasing. The average angular velocity of gratings was $73.1 \mathrm{dps}$. 


\section{Results}

We examined the effect on performance of changes in direction of motion caused by collisions with boundaries. We refer to such changes in direction of motion as bounces. If bounces attract attention, bounces of the nonqueried target should draw attention away from the queried target and decrease performance. We might also expect that bounces of the queried target should improve performance through increased attention to that object. This might not be evident in the data, however, if the effect is counteracted by any unknown deleterious effects of motion changes on orientation perception. If, for instance, the queried target passes back near its previous path, it may be masked by its own previous states. Another possibility is that attention may be biased after a direction change toward motion characteristics and away from orientation, the critical feature for the task. Attention might also be directed toward possible causes for the direction change, such as a search for nearby objects or boundaries. These are all effects of bounces on perception of the object that bounced. To examine only the attention-attracting effects of bounces, we tested the effect of a bounce in the nonqueried target on errors in reporting the queried target.

To examine the effect of recent nonqueried-target bounces, we examined the effect of bounces that occurred at different intervals before the end of the trial. Five in- tervals were used: 0-200, 201-400, 401-600, 601-800, and $801-1,000$ msec since the most recent bounce (see Figure 3). A sixth category contained trials for which no bounces occurred in the nonqueried target within the last $1,000 \mathrm{msec}$. We use the term error to refer to the magnitude of the error (absolute errors). Compared with trials in the sixth category, bounces of the nonqueried target in the last $200 \mathrm{msec}$ of the trial were associated with significantly greater errors (30.05 deg vs. 35.14 deg, respectively) $[t(1216)=2.67, p=.0078]$. This suggests that these irrelevant bounces attracted attention away from the queried target.

Bounces in none of the other four time windows produced significantly different accuracy compared with the baseline no-bounce trials [201-400 $\mathrm{msec}, t(1221)=$ $-0.49, p=.62 ; 401-600 \mathrm{msec}, t(1250)=0.06, p=$ $.95 ; 601-800 \mathrm{msec}, t(1227)=-0.04, p=.97 ; 801-$ $1,000 \mathrm{msec}, t(1180)=0.49, p=.62]$.

Overall, the mean number of bounces in the last $1,000 \mathrm{msec}$ of the trial was 0.63 , with $47 \%$ of trials containing no bounces in the last $1,000 \mathrm{msec}$ of the trial, $43 \%$ containing one bounce, $10 \%$ containing two bounces, and less than $1 \%$ containing three bounces. Trials were grouped according to the time of the last bounce, but some trials contained more than one bounce during the last second and there was thus a tendency for more bounces to have

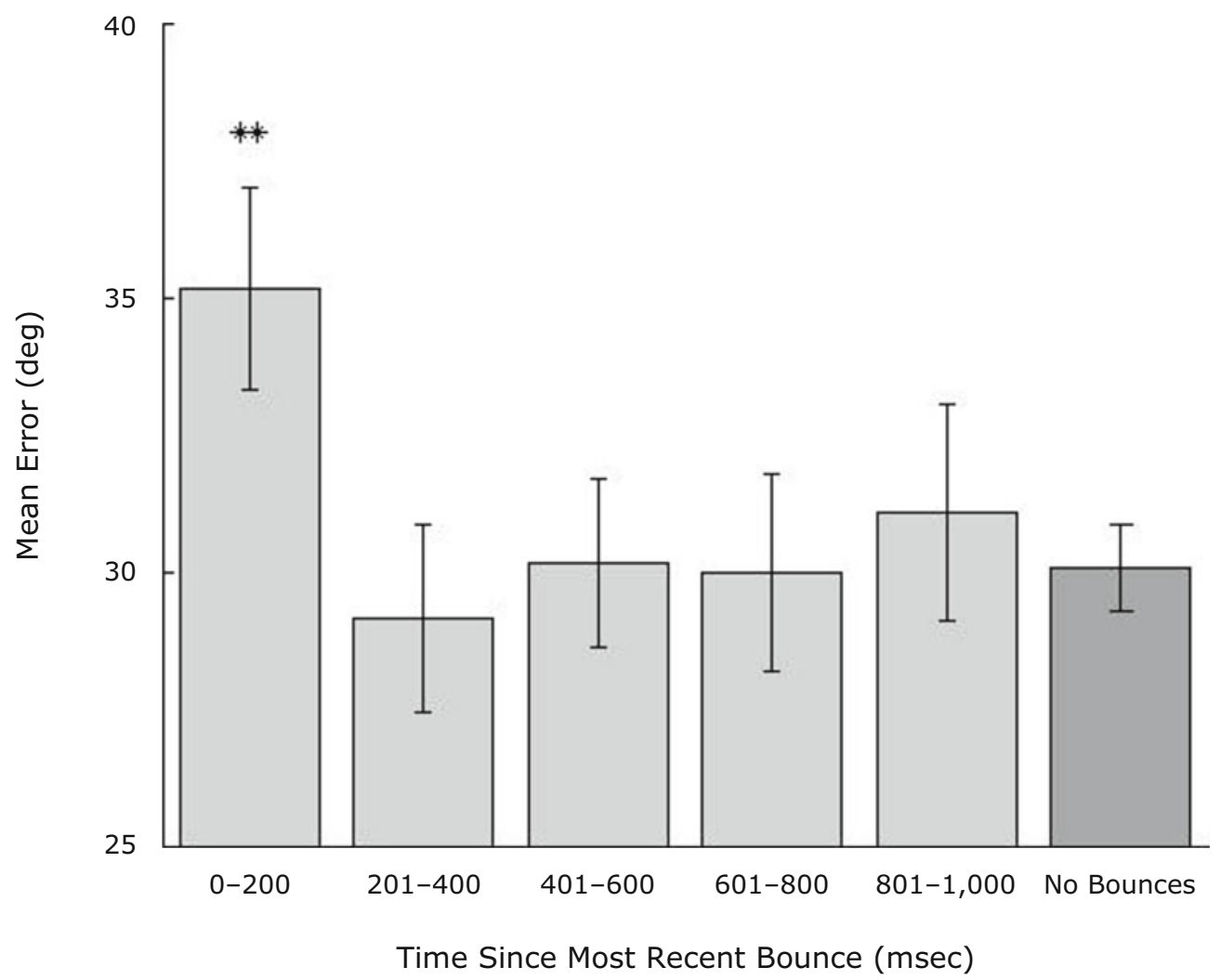

Figure 3. Mean absolute error magnitudes from Experiment 1. A bounce of the nonqueried target was detrimental to performance if it occurred within the last $200 \mathrm{msec}$ of the trial, suggesting that attention had been attracted away from the queried target. Error bars show $1 S E$ across participants. The rightmost bar shows trials in which no bounces occurred during the last 1,000 msec of the trial. 
occurred in those cases in which the last bounce was very recent. Trials in which the most recent bounce occurred within the last $200 \mathrm{msec}$ contained a mean of 1.30 bounces during the last second of the trial. The mean numbers of bounces were $1.36,1.18,1.13$, and 1.02 for the 201-400, 401-600, 601-800, and 801-1,000 msec windows, respectively. One possibility is that errors seen in the $0-200 \mathrm{msec}$ time window were exacerbated by the accumulated effects of multiple bounces in the last second rather than bounce recency per se. This is highly unlikely, however, given that there were more bounces for trials in which the most recent bounce occurred between 201 and $400 \mathrm{msec}$ before the end of the trial than in those in which the most recent bounce occurred in the $0-200 \mathrm{msec}$ window. If the number of bounces was driving the effect on performance, we would expect worse performance in the $201-400 \mathrm{msec}$ window than in the $0-200 \mathrm{msec}$ window, but we found the opposite. To further address this issue, we calculated mean errors for those $42 \%$ of trials in which only one bounce occurred during the last second of the trial. We categorized these trials according to when this bounce occurred, using the same five time windows as before. Mean errors were 33.25, $28.41,29.94,29.40$, and $30.58 \mathrm{deg}$ for the $0-200,201-$ $400,401-600,601-800$, and 801-1,000 msec windows, respectively. Even in this diminished data set, there was a tendency, albeit nonsignificant, for errors to be greatest for trials with a bounce in the last $200 \mathrm{msec}$ of the trial.

A direction change in the queried target may have yielded multiple and possibly opposing effects on performance as described in the results section for Experiment 1. Nevertheless, upon examination of the error (as we did for the nonqueried target), there was no significant effect of whether the queried target bounced recently $[0-200 \mathrm{msec}$, mean error $=29.60 \mathrm{deg}, t(1228)=-0.72, p=.47 ; 201-$ $400 \mathrm{msec}$, mean error $=30.74 \mathrm{deg}, t(1255)=-0.37, p=$ $.71 ; 401-600 \mathrm{msec}$, mean error $=32.36 \mathrm{deg}, t(1232)=$ $0.78, p=.44 ; 601-800 \mathrm{msec}$, mean error $=32.11 \mathrm{deg}$, $t(1250)=0.79, p=.43 ; 801-1,000 \mathrm{msec}$, mean error $=$ $29.26 \mathrm{deg}, t(1198)=-0.42, p=.68$; no bounce, mean error $=30.72 \mathrm{deg}]$.

In addition to examining the effect of bounces (a subset of the changes in direction that occurred), we examined the effects of changes in speed. Perception of acceleration or change in speed is limited to low temporal frequencies of speed variation, suggesting that the perception of speed may be the outcome of averaging motion signals over more than $100 \mathrm{msec}$ (Gottsdanker, 1956; Werkhoven, Snippe, \& Toet, 1992). Before further analysis, therefore, we smoothed the speed profile for each trial using a sliding rectangular 120 -msec window. There was no significant correlation between error and the resulting mean smoothed speed over the last $200 \mathrm{msec}$ of the trial $[r(2098)=.03, p=.25]$. Nor was there any significant relationship between error and the maximum smoothed speed over the last $200 \mathrm{msec}$ of the trial $[r(2098)=.03$, $p=.24]$ or for minimum smoothed speed over that period $[r(2098)=.03, p=.25]$.

We also examined the effect of change in speed on performance. As mentioned above, gratings moved with one of two possible accelerations (3.20 or $\left.8.00 \mathrm{deg} / \mathrm{sec}^{2}\right)$ in both the horizontal and vertical direction. Accuracy was not significantly correlated with mean change in smoothed absolute speed between successive frames over the last $200 \mathrm{msec}$ of the trial $[r(2098)=.002, p=.92]$, with maximum frame-to-frame change in smoothed speed over that period $[r(2098)=-.005, p=.82]$, or with minimum frame-to-frame change over that period $[r(2098)=.01$, $p=.65]$. Although this suggests speed changes did not attract attention, more systematic investigation or other experimental circumstances may show an effect.

Alvarez and Cavanagh (2005) found evidence that tracking resources in the left hemifield and those in the right hemifield were completely independent. Here there was only a very small and nonsignificant trend for an across-hemifield advantage: the mean error in the group with two targets in the same hemifield was $31.6 \mathrm{deg}$, and the mean error in the group with each target in a different hemifield was $30.2 \mathrm{deg}[t(19)=1.04, p=.31]$.

In Experiment 1, the boundaries that caused the bounces were not visible. In Experiment 2, the boundary boxes were made visible in order to investigate the role of visible causes of bounces.

\section{EXPERIMENT 2}

\section{Method}

Experiment 2 was identical to Experiment 1, except that the boundary boxes were made visible by drawing them in black lines, as shown in Figure 2.

Observers. Observers were 10 psychology staff members and 10 postgraduate students at Bristol University. Eighteen were naive to the purpose of the experiment, 10 had previously participated in Experiment 1, and 16 were nonexperts in participating in visual psychophysical experiments. All had normal or corrected-to-normal vision.

\section{Results}

We conducted the same analyses as those performed in Experiment 1 to assess whether bounces of the nonqueried target affected errors. As in Experiment 1, bounces were defined as changes in direction of motion caused by the boundary boxes, which were invisible in Experiment 1. In Experiment 2, effects of bounces in the nonqueried target at various times before the end of the trial showed no significant effects $[0-200 \mathrm{msec}, t(1250)=0.37, p=.71$; $201-400 \mathrm{msec}, t(1268)=0.73, p=.46 ; 401-600 \mathrm{msec}$, $t(1214)=-0.24, p=.81 ; 601-800 \mathrm{msec}, t(1216)=$ $-0.14, p=.89 ; 801-1,000 \mathrm{msec}, t(1190)=1.37, p=$ .17], as depicted in Figure 4. The only difference between Experiments 1 and 2 was that the box boundaries were visible in Experiment 2. Thus, it appears likely that the boxes rendered bounce events more predictable and thereby eliminated the attraction of attention to bounce events. We compared the effect of bounces of the nonqueried target in the last $200 \mathrm{msec}$ of the trial across Experiments 1 and 2 . To do this, we compared errors in the 0-200 msec bounce category after normalizing them by subtracting individuals' mean errors in the no-bounce (in the last $1,000 \mathrm{msec}$ ) baseline condition and then dividing by each individual's standard deviation of errors. The effect of these 0-200 msec nonqueried target bounces 


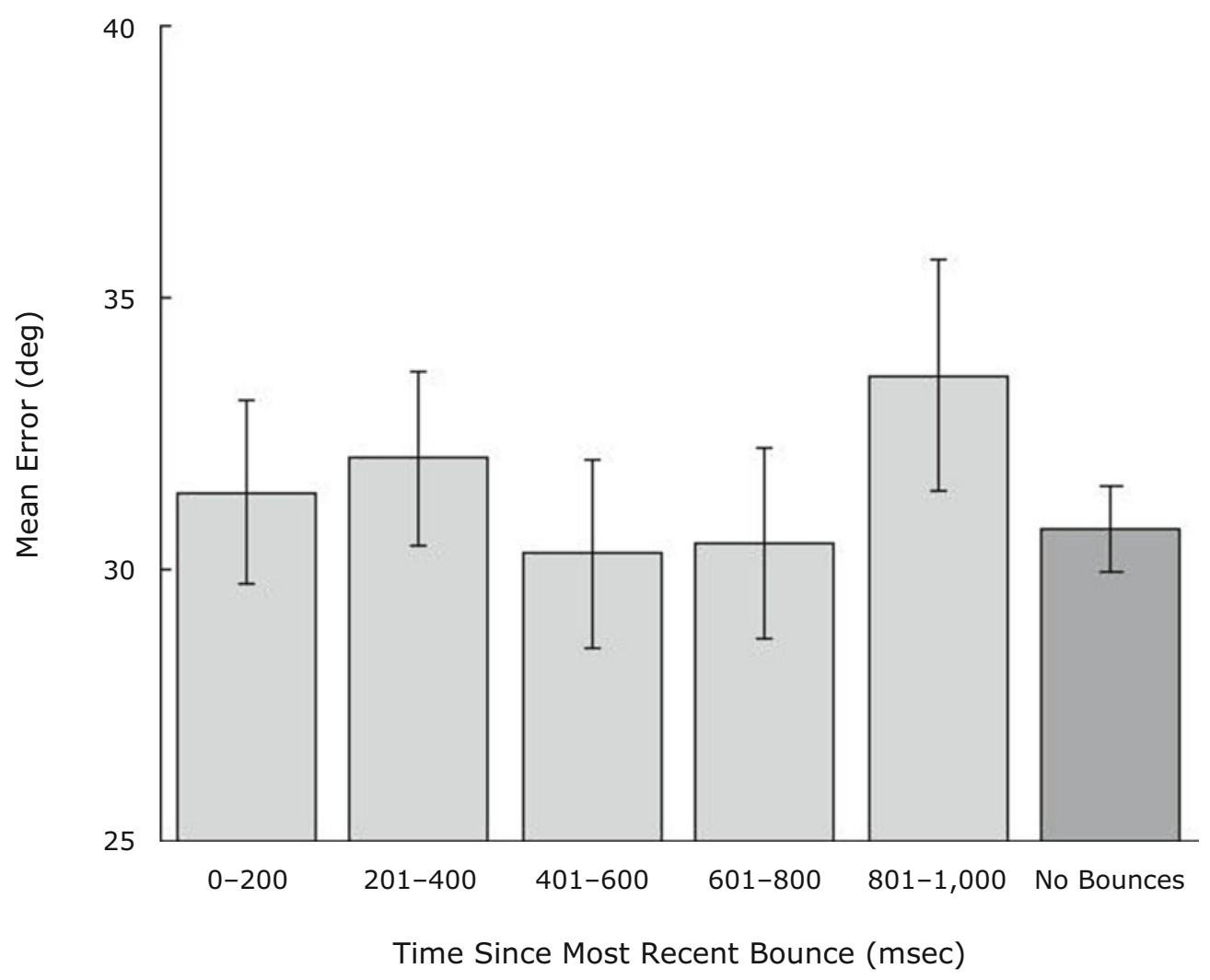

Figure 4. Mean absolute error magnitudes from Experiment 2. Bounces of the nonqueried target had no significant effect on performance. Error bars show $1 S E$ across participants. The rightmost bar shows trials where no bounces occurred during the last 1,000 msec of the trial.

on normalized error was significantly greater in Experiment 1 than in Experiment $2[t(459)=1.99, p=.047]$.

As in Experiment 1, bounces of the queried target in Experiment 2 had no significant effect on performance [0$200 \mathrm{msec}$, mean error $=31.40 \mathrm{deg}, t(1260)=0.31, p=$ $.76 ; 201-400 \mathrm{msec}$, mean error $=32.02 \mathrm{deg}, t(1248)=$ $1.21, p=.23 ; 401-600 \mathrm{msec}$, mean error $=30.27 \mathrm{deg}$, $t(1249)=1.93, p=.054 ; 601-800 \mathrm{msec}$, mean error $=$ $30.47 \mathrm{deg}, t(1240)=-0.12, p=.90 ; 801-1,000 \mathrm{msec}$, mean error $=33.55 \mathrm{deg}, t(1233)=-0.006, p=.995$; no bounce, mean error $=30.74 \mathrm{deg}]$.

One might worry that the presence of the boundary lines in Experiment 2 could affect visibility of the targets' orientations, but we found that overall orientation accuracy was similar between Experiment 1 (mean error $=$ $30.8 \mathrm{deg})$ and Experiment $2($ mean error $=31.3 \mathrm{deg})$ $[t(19)=0.29, p=.77]$.

In Experiment 2, there was again no significant correlation between error and mean smoothed speed over the last $200 \mathrm{msec}$ of the trial $[r(2098)=.004, p=.86]$, maximum smoothed speed over that period $[r(2098)=$ $.004, p=.86]$, or minimum smoothed speed over that pe$\operatorname{riod}[r(2098)=.004, p=.84]$. These three correlations between speed and error were highly similar because the three aspects of speed were each highly correlated with one another $(r>.99, p<.001$ in each case). Similarly, accuracy did not correlate significantly with change in smoothed absolute speed over the last $200 \mathrm{msec}$ of the trial $[r(2098)=-.01, p=.66]$, with maximum change in smoothed speed over that period $[r(2098)=-.02, p=$ $.40]$, or with minimum change in smoothed speed over that period $[r(2098)=-.001, p=.95]$.

The data from Experiment 2 support Alvarez and $\mathrm{Ca}-$ vanagh's (2005) hemifield-independence hypothesis more strongly than do the data from Experiment 1. In Experiment 2, the mean error when the targets were in the same hemifield was $33.6 \mathrm{deg}$, compared with $30.1 \mathrm{deg}$ when the targets were in different hemifields $[t(19)=4.02, p<$ $.01]$. Whether this 3 -deg difference in average error is the level expected from hemifield independence is uncertain, however. Alvarez and Cavanagh asserted that the hemifields were fully independent, meaning that performance in tracking two targets should be no worse than if only one target had been tracked. The level of performance when tracking only one target is unknown for this experiment, since it was not tested.

\section{GENERAL DISCUSSION}

In Experiment 1, recent bounces off boundary walls caused a decrement in performance when they occurred in the nonqueried target. We suggest that this was due to attention moving from the queried target to the nonqueried target. The effect was evident when the most recent 
bounce occurred in the $200 \mathrm{msec}$ preceding the end of the trial. The 200-msec time period is comparable to that of the effect of explicit cues in conventional attentional cuing studies, where processing benefits are typically seen for stimuli presented 80-300 msec after cuing (Nakayama \& Mackeben, 1989; Posner, 1980). When the boundary walls were visible in Experiment 2, the effect of these bounces disappeared, suggesting that it was the unexpected or unexplained nature of the direction changes that attracted attention.

Luminance changes usually co-occur with motion changes and can themselves capture attention (Christ \& Abrams, 2008; Enns et al., 2001; Ludwig, Ranson, \& Gilchrist, 2008). Here luminance changes occurred throughout because of the rotation and motion of the gratings. Effects of luminance changes may have interacted with those of bounces, but this cannot explain the elimination of the effect seen in Experiment 2, in which luminance characteristics were identical. Bounce events are associated with those positions on the screen farthest from the center of the boundary boxes, hence, it is possible that the presence of gratings in these locations attracted attention. Bounce events may also be associated with certain speed profiles, since very slowly moving objects, for instance, are likely to experience fewer collisions. However, neither of these factors can explain the elimination of the effect observed when the boundaries were made visible.

The magnitude of the main effect observed here (a 5-deg increase in orientation error) is comparable to a previously reported cost of $5 \mathrm{deg}$ for monitoring the orientations of two objects rather than one (Howard \& Holcombe, 2008). Howard and Holcombe measured the cost of splitting attention between two targets rather than allocating full attention to one target. In the experiments reported here, bounces of the nonqueried target likely reduced attention to the queried target further, causing attention to favor the nonqueried target. The cost for reporting a nonfavored target out of two targets appears comparable to the cost of splitting attention between two targets versus allocating full attention to one.

Given our poor sensitivity to changes in motion (Gottsdanker, 1956; Watamaniuk \& Heinen, 2003; Werkhoven et al., 1992; but cf. Pazo-Alvarez, Amenedo, \& Cadaveira, 2004), it may be surprising that changes in motion direction should attract attention. Perhaps only large changes in motion direction attract attention. Tripathy and Barrett (Tripathy, 2003; Tripathy \& Barnett, 2003, 2004) found that splitting attention among targets severely impaired the ability to detect small changes in direction. However, Tripathy and colleagues have shown that when direction changes were larger (as bounces were here, on average), many targets could be monitored successfully for a motion change (Tripathy \& Levi, 2008; Tripathy, Narasimhan, \& Barrett, 2007). In other words, a larger motion change was needed when fewer attentional resources were available per target. An interesting possibility raised by the present research is that when deviations are large, the change in direction may attract attention and thus assist the simultaneous monitoring of multiple trajectories.
That motion bounces may attract attention has implications for displays with moving objects, such as multiple object tracking (MOT), since many MOT displays include frequent collisions (Alvarez \& Franconeri, 2007; Pylyshyn, 1989; Pylyshyn \& Storm, 1988; Scholl \& Pylyshyn, 1999; Yantis, 1992). Recently, Iordanescu, Grabowecky, and Suzuki (2009) showed that observers were better at reporting positions of targets when they were near distractors, and it seems possible that collisions could have contributed to this result.

The ability of bounces to attract attention may have contributed to the attention-attracting effect of the "jitter" motion used by Franconeri and Simons (2003); however, such motion appears to have produced inconsistent results in the literature. For instance, Hillstrom and Yantis (1994) and Yantis and Egeth (1999) failed to find attention capture in an irrelevant-feature search task using oscillating motion among stationary distractors. The parameters of oscillating movement may not always be sufficient to attract attention for three reasons. First, the range of oscillating motion is typically limited to around $0.5 \mathrm{deg}$, much less than we used here. Second, because of the jitter type of motion used, the objects may simply have been perceived to possess positional imprecision over time rather than a persistent translational trajectory. Third, less predictable changes in motion may be required to attract attention.

Our findings are consistent with an account in which changes in direction of motion, when less expected, alert the observer to the possible presence of a previously undetected object with which a collision has occurred. New objects have been suggested to capture attention even in the absence of luminance changes (Yantis \& Hillstrom, 1994). Perhaps, then, these bounces attract attention because they signal the presence of a new object-in this case, the invisible box boundary.

Previous work has found that the sudden onset of motion can attract attention (Abrams \& Christ, 2003, 2005a, 2005b, 2006). Motion onsets can be considered a special case of change in motion - namely, a change from no motion to some motion. There are two reasons to suggest that whatever mechanism is responsible for this motion-onset effect may also be involved in the effect seen for unexpected bounces. First, motion onsets and unexpected bounces are both types of change in motion. Second, bounces attracted attention here only when they were unexpected, and motion-onset displays have typically used unexpected motion onsets or onsets with no visible cause.

Changes in motion can take the form of changes in direction or changes in speed. Motion onsets are also a special case of change in speed from no speed to some speed, which may contribute to their attention-attracting effect. Our analysis does not exclude the possibility of a further attentional benefit from speed changes. One might argue that all changes in motion are potential signs of animacy or important physical and social changes, such as people or animals quickening or slowing pace. More studies are needed to test this.

Might observers have realized the importance of the direction changes to the present experiments and directed 
extra attention to them? This is unlikely for several reasons. Most of the observers were naive to the purpose of the experiments, and none of the observers reported guessing the purpose of the experiment when it was explained to them afterward. From the perspective of the observers, there were many plausible purposes for the experiment, such as to investigate the effect of orientation, of the rate of orientation change, of reversals in rotation, or of the relative orientation of any combination of the four gratings. Other possible purposes included measurement of the effect of which quadrants contained the targets, of the relative positions of the four gratings, or indeed of the length of the trial and timing of various events during the trial.

We used a 300-msec delay between stimulus offset and postcue here to reduce the possibility of observers relying on high-fidelity, short-lived iconic memory. At shorter delays, observers do appear to use such memory for certain attentionally demanding tasks. For instance, Narasimhan, Tripathy, and Barrett (2009) saw thresholds rise sharply in a trajectory-deviation detection task when a delay of as little as $100 \mathrm{msec}$ was introduced between the two halves of the trajectory that were presented for comparison. If this memory survived our 300-msec delay in some weak form and observers used it, the memory trace must have been fragile enough to be affected by a bounce event in the nonqueried target. Perhaps nonqueried target bounces attracted attention away from the queried target for long enough to cause the trace to decay substantially further before the report was made.

The ability of changes in motion direction to attract attention may play an important role in our awareness of situations with multiple moving objects. Motion changes can signal important new information about the world, about the intentions of other creatures, and about forces acting on our immediate surroundings. In the modern world, awareness of motion changes may be critical in situations such as playing on the sports field, walking or driving on a busy street, and monitoring closed-circuit television.

\section{AUTHOR NOTE}

This research was supported by an EPSRC Grant to C.J.H. and by a Discovery Grant from the Australian Research Council to A.O.H. We thank Srimant Tripathy for his useful comments on the data presented here. Correspondence concerning this article should be addressed to C. J. Howard, School of Psychology, University of Sydney, Brennan MacCallum Bldg. A18, Sydney, NSW 2006, Australia (e-mail: cjhoward@ sydney.edu.au).

\section{REFERENCES}

Abrams, R. A., \& Christ, S. E. (2003). Motion onset captures attention. Psychological Science, 14, 427-432.

Abrams, R. A., \& Christ, S. E. (2005a). Onset but not offset of irrelevant motion disrupts inhibition of return. Perception \& Psychophysics, 67, 1460-1467.

Abrams, R. A., \& Christ, S. E. (2005b). The onset of receding motion captures attention: Comment on Franconeri and Simons (2003). Perception \& Psychophysics, 67, 219-223.

Abrams, R. A., \& Christ, S. E. (2006). Motion onset captures attention: A rejoinder to Franconeri and Simons (2005). Perception \& Psychophysics, 68, 114-117.

Alvarez, G. A., \& Cavanagh, P. (2005). Independent resources for attentional tracking in the left and right visual hemifields. Psychological Science, 16, 637-643.

Alvarez, G. A., \& Franconeri, S. L. (2007). How many objects can you track? Evidence for a resource-limited attentive tracking mechanism. Journal of Vision, 7 (13, Art. 14), 1-10.

Ansorge, U., Scharlau, I., \& LABUdDa, K. (2006). Visual search for a motion singleton among coherently moving distractors. Psychological Research, 70, 103-116.

Averbach, E., \& Coriell, A. S. (1961). Short-term memory in vision. Bell System Technical Journal, 40, 309-328.

Christ, S. E., \& Abrams, R. A. (2008). The attentional influence of new objects and new motion. Journal of Vision, 8 (3, Art. 27), 27, 1-8.

Coltheart, M., Lea, C. D., \& Thompson, K. (1974). In defence of iconic memory. Quarterly Journal of Experimental Psychology, 26, 633-641.

Enns, J. T., Austen, E. L., Di Lollo, V., Rauschenberger, R., \& YANTIS, S. (2001). New objects dominate luminance transients in setting attentional priority. Journal of Experimental Psychology: Human Perception \& Performance, 27, 1287-1302.

Franconeri, S. L., \& Simons, D. J. (2003). Moving and looming stimuli capture attention. Perception \& Psychophysics, 65, 999-1010.

Franconeri, S. L., \& Simons, D. J. (2005). The dynamic events that capture visual attention: A reply to Abrams and Christ (2005). Perception \& Psychophysics, 67, 962-966.

GotTSDANKER, R. M. (1956). The ability of human operators to detect acceleration of target motion. Psychological Bulletin, 53, 477-487.

Hillstrom, A. P., \& Yantis, S. (1994). Visual motion and attentional capture. Perception \& Psychophysics, 55, 399-411.

Horowitz, T. S., Wolfe, J. M., DiMase, J. S., \& Klieger, S. B. (2007). Visual search for type of motion is based on simple motion primitives. Perception, 36, 1624-1634.

HorstmanN, G. (2005). Attentional capture by an unannounced color singleton depends on expectation discrepancy. Journal of Experimental Psychology: Human Perception \& Performance, 31, 1039-1060.

Howard, C. J., \& Holcombe, A. O. (2008). Tracking the changing features of multiple objects: Progressively poorer perceptual precision and progressively greater perceptual lag. Vision Research, 48, 11641180 .

Iordanescu, L., Grabowecky, M., \& Suzuki, S. (2009). Demandbased dynamic distribution of attention and monitoring of velocities during multiple-object tracking. Journal of Vision, 9 (4, Art.1), 1-12.

Johnson, J. D., Hutchison, K. A., \& Neill, W. T. (2001). Attentional capture by irrelevant color singletons. Journal of Experimental Psychology: Human Perception \& Performance, 27, 841-847.

LudwiG, C. J. H., Ranson, A., \& Gilchrist, I. D. (2008). Oculomotor capture by transient events: A comparison of abrupt onsets, offsets, motion, and flicker. Journal of Vision, 8 (14, Art. 11), 1-16.

Micнотте, A. (1963). The perception of causality (T. R. Miles \& E. Miles, Trans.). New York: Basic Books.

Nakayama, K., \& Mackeben, M. (1989). Sustained and transient components of focal visual attention. Vision Research, 29, 1631-1647.

Narasimhan, S., Tripathy, S. P., \& Barrett, B. T. (2009). Loss of positional information when tracking multiple moving dots: The role of visual memory. Vision Research, 49, 10-27.

Pazo-Alvarez, P., Amenedo, E., \& Cadaveira, F. (2004). Automatic detection of motion direction changes in the human brain. European Journal of Neuroscience, 19, 1978-1986.

Posner, M. I. (1980). Orienting of attention. Quarterly Journal of Experimental Psychology, 32, 3-25.

Pylyshyn, Z. W. (1989). The role of location indexes in spatial perception: A sketch of the fINST spatial-index model. Cognition, 32, 65-97.

PyLYShyn, Z. W., \& STORM, R. W. (1988). Tracking multiple independent targets: Evidence for a parallel tracking mechanism. Spatial Vision, 3, 179-197.

Rushton, S. K., Bradshaw, M. F., \& Warren, P. A. (2007). The pop out of scene-relative object movement against retinal motion due to self-movement. Cognition, 105, 237-245.

Scholl, B. J., \& Pylyshyn, Z. W. (1999). Tracking multiple items through occlusion: Clues to visual objecthood. Cognitive Psychology, 38, 259-290.

Shooner, C., Tripathy, S. P., Bedell, H. E., \& Ögmen, H. (2010). 
High-capacity, transient retention of direction-of-motion information for multiple moving objects. Journal of Vision, 10 (6, Art. 8), 1-20.

SPERLING, G. (1960). The information available in brief visual presentations. Psychological Monographs, 74, 1-29.

Straw, A. D. (2008). Vision egg: An open-source library for realtime visual stimulus generation. Frontiers in Neuroinformatics, 2 (Art. 4), 1-10. doi:10.3389/neuro.11.004.2008

TRIPATHY, S. P. (2003). Severe loss of positional information when tracking multiple dots. Journal of Vision, 3 (9, Art. 340a).

Tripathy, S. P., \& Barrett, B. T. (2003). Last but not least: Gross misperceptions in the perceived trajectories of moving dots. Perception, 32, 1403-1408.

TRIPATHY, S. P., \& Barrett, B. T. (2004). Severe loss of positional information when detecting deviations in multiple trajectories. Journal of Vision, 4, 1020-1043.

Tripathy, S. P., \& Levi, D. M. (2008). On the effective number of tracked trajectories in amblyopic human vision. Journal of Vision, 8 (4, Art. 8), 1-22.

Tripathy, S. P., Narasimhan, S., \& Barrett, B. T. (2007). On the effective number of tracked trajectories in normal human vision. Journal of Vision, 7 (6, Art. 2), 1-18.

von Mühlenen, A., Rempel, M. I., \& EnNs, J. T. (2005). Unique tem- poral change is the key to attentional capture. Psychological Science, 16, 979-986.

Watamaniuk, S. N. J., \& Heinen, S. J. (2003). Perceptual and oculomotor evidence of limitations on processing accelerating motion. Journal of Vision, 3, 698-709.

Werkhoven, P., Snippe, H. P., \& Toet, A. (1992). Visual processing of optic acceleration. Vision Research, 32, 2313-2329.

YANTIS, S. (1992). Multielement visual tracking: Attention and perceptual organization. Cognitive Psychology, 24, 295-340.

Yantis, S., \& EgEth, H. E. (1999). On the distinction between visual salience and stimulus-driven attentional capture. Journal of Experimental Psychology: Human Perception \& Performance, 25, 661-676.

YANTIS, S., \& Hillstrom, A. P. (1994). Stimulus-driven attentional capture: Evidence from equiluminant visual objects. Journal of Experimental Psychology: Human Perception \& Performance, 20, 95-107.

YANTIS, S., \& Jonides, J. (1984). Abrupt visual onsets and selective attention: Evidence from visual search. Journal of Experimental Psychology: Human Perception \& Performance, 10, 601-621.

(Manuscript received March 2, 2009;

revision accepted for publication June 4, 2010.) 